\title{
Using IEC 61850 for Switchgear Online Condition Monitoring
}

\author{
Jun Jiang \\ Electrical Engineering Department \\ Shandong Polytechnic \\ Jinan, China \\ E-mail: Jiang_jun96@163.com
}

\begin{abstract}
The online condition monitoring system diagnoses power equipment health status. And now it is one of the major issues to improve the reliability of the power system by preventing a potential failure in advance. Since there are too many different condition monitoring techniques are used, they need to be standardized in IEC 61850. Take gas insulated switchgear (GIS) as a example, this paper studied the condition monitoring diagnosis informatiom model and system integration. GIS is a compact metal encapsulated switchgear consisting of circuit-breakers and disconnectors. GIS condition monitoring system acquires condition data from sensors that are placed in the components. Based on IEC 61850, the modeling method and the application of logical node are standard. And the format of spectra data also is standard. And the paper also standards configuration of intelligent electronic device (IED), conformance testing of IED. The practical application shows that the standardization for switchgear condition monitoring is beneficial for access to the remote center. And the standard based on IEC 61850 is benefit for condition monitoring system intergration.
\end{abstract}

Keywords-online condition monitoring; condition monitoring diagnosis and analysis; system integration; information model; IEC 61850

\section{INTRODUCTION}

In power system, gas insulated switchgears (GIS) are the combination of circuit breakers, disconnector switches, earthing switches and instrument transformers [1]. The compartments contain the insulating medium, such as SF6 or gas mixture [2-3]. GIS is often used where space is limited, for example, in city buildings, on roofs. As a result, GIS offers outstanding reliability, operational safety and environmental compatibility. So, it is widely used in substations. To reduce the cost of maintenance, conditionbased maintenance is required.

For the complex sealed equipment, it is difficult to diagnose internal conditions from outside, and thus various sensors have been proposed for fault detection. As one of the main techniques of monitoring insulation condition, partial discharge in recent years is the most widely used. Some method such as high frequency detection and ultrasonic detection based on decomposition gases generated by partial discharge are commonly used. Strathclyde University of UK introduced a linear relationship between the square of the energy of the high frequency signal and the square of the discharge of the high frequency signal in refrence [4-5]. The first special high frequency monitoring device was invented in 1986, and installed in Scotland Torness nuclear power plant [6]. In Chian, Xi'an Jiao Tong University has also studied the method [7-8]. Ultrasonic testing technology in China also has a wide range of applications [9-10], such as Guangdong], Shandong, Yunnan and other places have a corresponding application case.

With the application of condition monitoring of GIS, some problems are also appeared. It is very difficult for condition monitoring from different vendor to connect with others for sharing information. Vast amounts of data, power utility is notoriously difficult to comprehensive application for GIS pre-fault comprehensive application. In this context, the power grid utility of China proposes a standardization information application based on IEC 61850 for GIS on-line condition monitoring device. In this application, the information model and the information model of intelligent electronic device (IED) are standard based on IEC 61850. And all the IED for online condition monitoring are linked to the master station in control.

\section{GIS ONLINE CONDITION MONITORING SYSTEM}

\section{A. GIS Overview}

GIS is a kind of metal enclosed switchgear. A GIS consists of several components as circuit breakers, disconnector switches, earthing switches and instrument transformers. All the equipment of the electrical switchgear are enclosed by gas tight metal enclosure and SF6 gas is used as insulation between live parts of the equipment and earthed metal enclosure. GIS is safe to open under normal load current, while protective devices are safe to open under fault current. And GIS is available from $12 \mathrm{KV}$ system to $800 \mathrm{KV}$ system.

\section{B. System Structure}

In most cases a GIS Monitoring system is installed in parallel to the control system. IEDs for different primary equipment from different vendors are connected to a network and share information with the master station in the control center. It implements the entire power grid primary equipment online condition monitoring, so as to improve the level of fault diagnosis. The system structure is illustrated in Fig.1. 


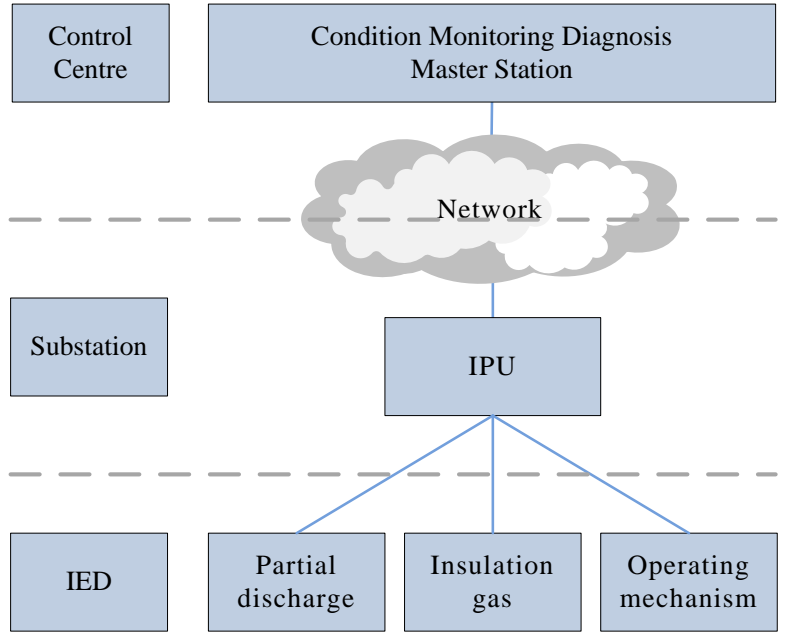

Figure 1. Structure of condition monitoring diagnosis system

As Fig. 1 shows, online condition monitoring diagnosis (CMD) system can be divided into three levels: master station, integrated process unit (IPU), filed IED. The master station in the control centre acquires condition data from IED or sensors that are installed at the different GIS equipments. Applications of master station include equipment supervision, maintenance planning support, and configuration management. IPU exchanges data such as measurement, alarm, spectra with CMD master station or other computers in the control centre via a wide network. IEDs and all kinds of sensors measures various information from a target equipment, and check data is within the normal range or not.

The basic function of the master station in the remote center include:

(1) display of the real-time operation data;

(2) query and display historical operation data;

(3) fault diagnosis for the GIS equipment;

(4) discharge spectra data display.

\section{Partial Discharge Supervision.}

The most common internal fault of GIS is the insulation fault. When the internal insulation failure occurs in the GIS, the partial discharge can be produced, and the SF6 gas is decomposed, which leads to the serious damage of the insulation material. According to these physical and chemical changes, the local discharge can be detected. At present, the method of electric method and vibration method. The widely used method includes electrical method and vibration method.

Electrical method has the external complex electrode method, inner complex electrode method, ultra high frequency method. And the ultra high frequency method is widely used method. GIS partial discharge can be judged by the detection of the high frequency electromagnetic wave signal $(0.3 \mathrm{GHz} \sim 3 \mathrm{GHz})$. This method can be used for on-line monitoring, pattern recognition and fault location.

Vibration method is used to detect the acoustic wave and ultrasonic wave generated by partial discharge. This method uses the sensors installed in the GIS to detect internal defects discharge. Acoustic wave $1 \mathrm{kHz} \sim 20 \mathrm{kHz}$ and Ultrasonic $1 \mathrm{kHz} 20 \mathrm{kHz}$ are used for detecion.

\section{Insulation Gas Supervision}

Insulation gas supervision can be used for any enclosed insulated gas in a substation. The goal of supervision is to detect early gas leakage and arrange equipment maintenance. It is use the pressure and sensitivity of the gas to detect leaks. Gas sensor measures the pressure and temperature inside a gas compartment of the GIS. Density calculation is performed by the sensor itself or by the IED's.

\section{E. Operating Mechanism.}

High voltage circuit breaker always uses the electromagnet at the first stage of the operation control components. So, dc electromagnetic coil current waveform can be used as the important basis of mechanical fault diagnosis. The open-brake coil and close-break coil current waveform use the way of fault wave record and store in spectral files.

\section{DATA MODELING}

\section{A. IEC 61850 Overview}

IEC 61850 series standard is the cooperation of Electric Power Research Institute (EPRI), Institute of Electrical and Electronics Engineers (IEEE) and the International Electrotechnical Commission (IEC). The purpose of IEC 61850 is creating a global network standards to solve the substation automation system network and communication.. Using the object-oriented modeling method and hierarchical distributed structure system, it standardizes the intelligent electronic device (IED) communication data and communication interface. Using extensible markup language (XML) based substation configuration language (SCL); it realizes the self-description of IED. IEC 61850 is the basis of realizing the interoperability and seamless integration for IED.

\section{B. Modeling method based on IEC 61850.}

IEC 61850 is used as the standard information model and information exchange model for power equipment condition monitoring diagnosis. Now, the basic ideas of IEC 61850 and modeling method have been widely used in the field of power system automation, such as substation automation, distribution automation[11-12], and so on. The special report of using IEC 61850 for condition monitoring diagnosis and analysis is formed.

Information model and modeling method are the core of the IEC 61850 standard. Using object oriented and virtualization method, IEC 61850 treats the actual physical device as a virtual device. The actual device is decomposed into Server, Logical Device (LD), Logical Node (LN), Data Object (DO), and Data Attribute (DA). The communication between the online monitoring device, the online monitoring IPU and the remote center master station system becomes the information exchange between the information objects.

The IED capability description (ICD) files use "one IED --- one Logical Device --- multi Logical Nodes". It means that one online monitoring device has one Logical Device and a number of application functional entities. And one IPU can connect with several online monitoring devices. 


\section{Logical Nodes}

The logical nodes defined in IEC 61850 can meet with a part of online condition monitoring functions. Some specific functions should be modeled as new logical nodes. GIS online condition monitoring should include the following logical nodes listed in Table 1 as necessary for a particular. Most of the logical nodes are defined in IEC 61850-7-4 secondary version. The new logical node TUHF defined as Fig.2.

TABLE I. LOGICAL NODES

\begin{tabular}{|c|c|c|}
\hline Name & Description & Extension \\
\hline TTMP & Temperature sensor & $7-4$ ed 2 \\
\hline THUM & Humidity sensor & $7-4$ ed 2 \\
\hline TPRS & Pressure sensor & $7-4$ ed 2 \\
\hline TCTR & Current transformer & $7-4$ ed 2 \\
\hline TVTR & Voltage transformer & $7-4$ ed 2 \\
\hline TANG & Angle & $7-4$ ed 2 \\
\hline TUHF & UHF Sensor & New \\
\hline SIMG & Insulation medium supervision (gas) & $7-4$ ed 2 \\
\hline SIML & Insulation medium supervision (liquid) & \\
\hline STMP & Temperature supervision & $7-4$ ed 2 \\
\hline SARC & Monitoring and diagnostics for arcs & $7-4$ ed 2 \\
\hline SCBR & Circuit breaker s upervision & $7-4$ ed 2 \\
\hline SOPM & $\begin{array}{l}\text { Supervision of operating } \\
\text { mechanism }\end{array}$ & $7-4$ ed 2 \\
\hline SPDC & $\begin{array}{l}\text { Monitoring and diagnostics for partial } \\
\text { discharges }\end{array}$ & $7-4$ ed 2 \\
\hline XCBR & Circuit breaker & $7-4$ ed 2 \\
\hline
\end{tabular}

\begin{tabular}{|c|c|c|c|}
\hline \multicolumn{4}{|c|}{ TUHF (Ultra High Frequency Sensor) } \\
\hline $\begin{array}{c}\text { Data object } \\
\text { name }\end{array}$ & $\begin{array}{l}\text { Common } \\
\text { data class }\end{array}$ & Explanation & $\mathrm{M} / \mathrm{O}$ \\
\hline LNName & & $\begin{array}{l}\text { The name shall be composed of the class } \\
\text { name, the LN-Prefix and LN-Instance-ID. }\end{array}$ & M \\
\hline \multicolumn{4}{|l|}{ Data objects } \\
\hline \multicolumn{4}{|l|}{ Description } \\
\hline EEName & DPL & equipment name plate & $\mathrm{O}$ \\
\hline \multicolumn{4}{|c|}{ Status information } \\
\hline EEHealth & ENS & External equipment health & $\mathrm{O}$ \\
\hline \multicolumn{4}{|c|}{ Measured and metered values } \\
\hline UhfSv & SAV & UHF signal & M \\
\hline
\end{tabular}

Figure 2. TUHF logical node

\section{Spectra Data.}

In GIS online condition monitoring, spectra data is a very important kind of data and it can directly reflect the GIS equipment working status, fault detection, and tracking latent fault. The partial discharge spectrum and the circuit breaker monitoring data are the two kinds of widely used spectra data.

The partial discharge spectra data is composed of a period of time of discharge intensity, frequency and phase. And it is focused on the relationship between the intensity of the discharge signal and the phase and frequency of the discharge signal. The discharge intensity refers to the discharge signal peak of the detection period. Discharge phase refers to the time occur in each cycle of the power frequency signal peak corresponding detection period. Discharge frequency refers to the various testing period, have different threshold intensity of discharge.

The circuit breaker monitoring spectrum data is acquired online monitoring device of waveform data, including breaking current waveform and closing coil current waveform, brake coil current waveform storage, motor current waveform and so on.

\section{IED TESTING}

\section{A. Device Verification Testing}

In order to standardize the parameters of GIS on-line monitoring IED, it is needed for verification testing. The testing also makes IEDs linking to the master station easily.

Verification Testing includes: sensitivity testing, dynamic range testing, alarm function testing, discharge testing, self detection function testing, and so on. To test the validity of different GIS partial discharge on-line monitoring device manufacturers, use a stable and adjustable analog signal for testing. Each IED manufacturers test the signal respectively. According to the result of partial discharge measurement, the test result comprehensive compare the sensor sensitivity and effectiveness of every manufacturers.

TABLE II. IEDS EQUIPPED RULE

\begin{tabular}{|c|c|}
\hline $\begin{array}{c}\text { Voltage } \\
\text { level }\end{array}$ & Equipped rules \\
\hline \multirow{2}{*}{$500 \mathrm{kV}$} & Priority to the use of the method of charging testing. \\
\hline & $\begin{array}{l}\text { Pilot installation partial discharge on-line monitoring } \\
\text { device. }\end{array}$ \\
\hline \multirow{2}{*}{$200 \mathrm{kV}$} & $\begin{array}{l}\text { Adopting the combination of charged and online way } \\
\text { to partial discharge test. }\end{array}$ \\
\hline & $\begin{array}{l}\text { Domestic manufacturers of equipment should install } \\
\text { partial discharge on-line monitoring device. }\end{array}$ \\
\hline \multirow{2}{*}{$100 \mathrm{kV}$} & Partial discharge test preferred charged test way. \\
\hline & $\begin{array}{l}\text { Important substation that supply power to the } \\
\text { important users should install partial discharge on } \\
\text { line monitoring device. }\end{array}$ \\
\hline
\end{tabular}




\section{B. Configuration of On-line Monitoring IED}

Considering different voltage GIS monitoring device security, effectiveness, reliability, necessity and economy, define different voltage GIS monitoring device configuration rules as shown in Table II.

\section{USE CASE STUdY}

A southern coastal city power supply company has installed GIS online condition monitoring devices, IPU in one substation and master station in the control center. Remote on-line condition monitoring for GIS equipment is realized.

In July 27, 2011, the partial discharge signal was detected at a line of the substation. At 18 o'clock, the amplitude and frequency of the discharge signal are obviously increased, and the adjacent sensor is also monitoring the obvious signal, and the development trend is enhanced as showed in Fig.3. The exclusion of interference and other factors, the online CMD system suggested testing equipment. July 30 , the engineer tested the line GIS No. 1612 by portable instrument. The results showed that the fault occurred at GIS A phase cable terminal. Partial discharge of the remote center to capture is recorded.

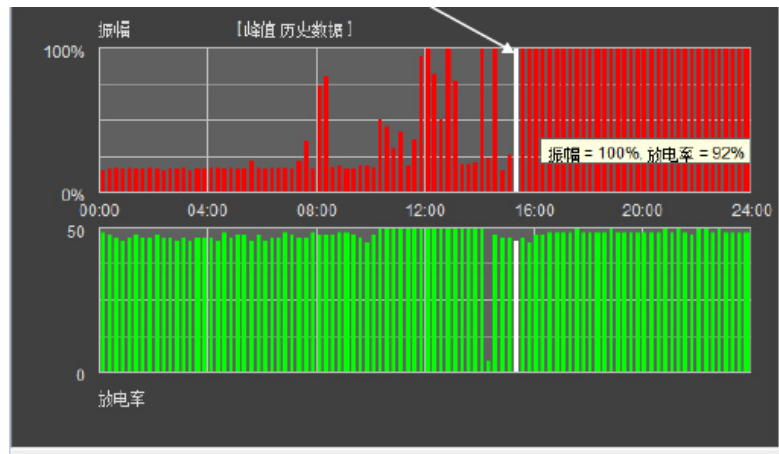

莎一个 后一个 历史天数据 on 27 Ju1 2011 [15:15]

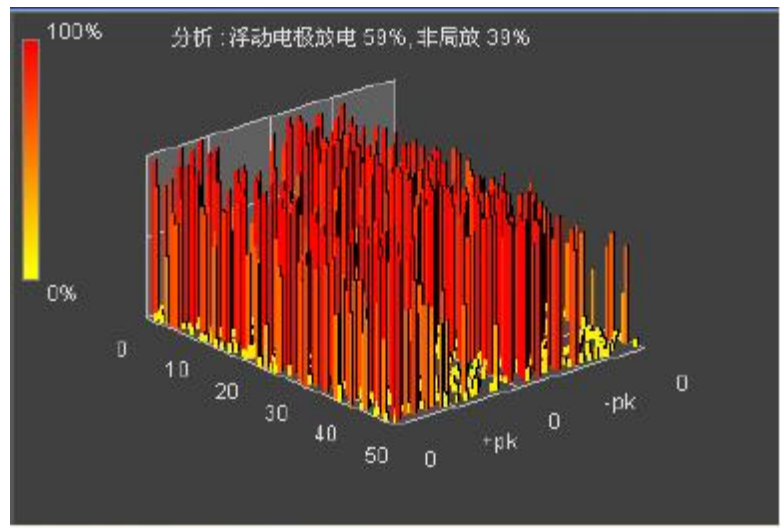

事件序号 130 记录 27 Jul 2011 at 15:06:56

Figure 3. 2011-7-27 Partial discharge detetced by the control centre

After ruled out the interference factors, such as field charged system suggested by test.On July 30, technical personnel using the portable instrument for couple OCU13 show high I line 1612 GIS equipment near interval and wire cable poisoned the activity test and positioning, the fault location in line show high I GIS A cable terminal.

After eliminating the interference and other factors, the system is recommended for the field live test. July 30, engineers use the I\# line high portable instrument near the couple OCU1-3 and shows that 1612 GIS equipment interval and the cable outlet of partial discharge activity test and positioning. And then engineers locate the fault in GIS and the fault is at a cable terminal head of I\# line.

In this case, the remote center master station use remote online monitoring data and comprehensive data analysis, find the problems quickly. And the master station also helps the engineer for field testing, to avoid the occurrence of the fault.

\section{CONCLUSIONS}

GIS online condition monitoring is an important content of power equipment condition monitoring system. Using remote online monitoring, it is to improve the comprehensive utilization of the monitoring data. And it also improves the level of the GIS equipment state overhaul, for early detection failures.

Using IEC 61850 modeling method is helpful for information model and information service model. The application of IEC 61850 improves data sharing online device, convenient for system integration.

\section{REFERENCES}

[1] Alexandra Von Meier (2013). Electrical Engineer 137A: Electric Power Systems. Lecture 14:Introduction to Protection Systems.

[2] Switchgear[EB/OL] https://en.wikipedia.org/wiki/Switchgear

[3] Power-system protection[EB/OL] https://en.wikipedia.org/ wiki/ Power-system_protection

[4] Hampton B F, Meats R J. Diagnostic measurements at UHF in gas insulated substations $[\mathrm{C}] / /$ Generation, Transmission and Distribution, IEE Proceedings C. IET, 1988, 135(2): 137-145.

[5] Pearson J S, Hampton B F, Sellars A G. A continuous UHF monitor for gas-insulated substations[J]. Electrical Insulation, IEEE Transactions on, 1991, 26(3): 469-478

[6] Judd M D, Yang L, Hunter I B B. Partial discharge monitoring of power transformers using UHF sensors. Part I: sensors and signal interpretation[J]. Electrical Insulation Magazine, IEEE, 2005, 21(2): 5-14.

[7] Xiaohui Z, Jinggang Y, Xiuli L U. Compa rative resea rch on curent pulse method and UHF measurements of partia 1 discharge in minera 1 oil[J]. High Voltage Engineer ing, 2008, 34(7): 14011404

[8] ZHAO X, YANG J, LU X, et al. Study of PD Location Based on the UHF Method[J]. High Voltage Apparatus, 2008, 4: 031.

[9] Yuan L, Jianshe X, Xiaohui Z. Ultra-Wide Band Technology in Data Acquisition System's Applied Research[J]. China New Telecommunications, 2008, 23: 018.

[10] Fang W, Daoyin Q, Yanjie Y. Wireless ther-moelectric temperature monitoring of substation based on infrared technology[J]. Electric Power Automation Equip-ment, 2011, 31(8): 135-138.

[11] Han Guozheng, Xu Bingyin , Suonan Jiale. IEC 61850-based feeder terminal unit modeling and mapping to IEC 60870-5-104[J] Power Delivery, IEEE Transactions on, 2012, 27(4): 2046-2053.

[12] Han Guozheng, $\mathrm{Xu}$ Bingyin. IEC 61850-based open communication system of advanced distribution automation[J]. Power System Technology, 2011, 35(4): 183-186. 